How to reference this article

Bartkowiak, J. (2020). La Medusa decapitata: la donna nel racconto ecfrastico La ripetizione di Andrea Camilleri e nel quadro La Vucciria di Renato Guttuso. Italica Wratislaviensia, 11(2), 55-72.

DOI: http://dx.doi.org/10.15804/IW.2020.11.2.4

Joanna Bartkowiak

Uniwersytet im. Adama Mickiewicza w Poznaniu, Polonia

joanna.bartkowiak@amu.edu.pl

ORCID: 0000-0003-1564-8670

\title{
LA MEDUSA DECAPITATA: \\ LA DONNA NEL RACCONTO ECFRASTICO LA RIPETIZIONE DI ANDREA CAMILLERI E NEL QUADRO LA VUCCIRIA DI RENATO GUTTUSO
}

\author{
THE DECAPITATED MEDUSA: THE WOMAN \\ IN THE EKPHRASTIC WORK LA RIPETIZIONE \\ BY ANDREA CAMILLERI AND IN THE PAINTING \\ LA VUCCIRIA BY RENATO GUTTUSO
}

\begin{abstract}
The main focus of this article is to find an image of the woman, provided by an ekphrastic work by Andrea Camilleri, La ripetizione, corresponding to a famous painting by Renato Guttuso, La Vucciria. The duality of the perception of the woman, in the rich and multi-millennial Sicilian culture, is reflected in the writer's narrative through a clash of roles and an inversion of the positions commonly assigned to individuals by society. The analysis highlights the mutual exchange of glazes, defined by Laura Mulvey as "male" and "female," and the installation within the framework of the observer reflected by the mirror, as proposed by Michel Foucault. The reflections of psychoanalysts, in particular Julia Kristeva and Melanie Klein, provide some explanations regarding the coexistence of the cult of the mother with the desire for matricide. According to these theories, the figure of the male child remains forever burdened by an inconsolable sense of guilt and frustration. Consequently, the male protagonists of Camilleri are tormented by a terror of and a disdain for the female body. In fact, their domination proves to be only illusory: the image of the woman is overwhelming and threatening like that of Trinacria, the mistress of Sicily. Finally, the male, fighting for his own identity, commits matricide and the Medusa is beheaded.
\end{abstract}

Keywords: portrait, woman, Camilleri, Guttuso, matricide 
$\mathrm{La}$ donna siciliana, a partire dal Medioevo, appare sempre nelle arti e nella poesia come un personaggio presente ma sfuggente ${ }^{1}$. Risalendo alla tradizione letteraria esistente, già alla corte di Federico II la sua rappresentazione sembra molto offuscata ${ }^{2}$. Parallelamente l'importanza e la posizione della donna nella società siciliana (anche se considerata "ambigua") è costituzionale poiché simbolicamente la Sicilia "è una donna": il suo volto femminile è quello della Medusa. La testa mostruosa della Medusa (la più famosa delle tre sorelle mitologiche, rappresenta la perversione intellettuale), fa parte del simbolo più antico della Sicilia: la Trinacria.

L'articolo propone uno studio del caso ${ }^{3}$ attraverso un' analisi comparativa riguardante due particolari opere di due autori siciliani: lo scrittore Andrea Camilleri (1925-2019) e il pittore Renato Guttuso (1911-1987). In particolare, il testo verterà sulla ricostruzione del ritratto letterario e artistico della donna nell'opera ecfrastica La ripetizione confrontandolo con il quadro La Vucciria. Le donne, come notato da Giuseppe Fabiano, "sono state un punto di riferimento importante" (Fabiano, 2019, p. 69) per Camilleri: il figlio unico ha "vissuto in una casa dove le donne della famiglia avevano un ruolo centrale" (ibid.). Lo dimostrano i romanzi dove la donna o le donne esercitano un ruolo cruciale o persino sono la chiave di tutta la narrazione: basta menzionare opere come La pensione Eva (2006), Il tailleur grigio (2008), Trilogia delle metamorfosi

${ }^{1}$ La sua immagine proviene soprattutto dalla mente, dalla memoria, troppo grande per gli occhi del poeta: "come pote si gran donna entrare per gli ochi miei che sì piccioli sone?" (Giacomo da Lentini, Or come pote sì gran donna entrare). A volte, la donna è proprietaria di poteri quasi soprannaturali: gli occhi che inducono ad innamorarsi con un solo sguardo: "Uno piasente sguardo coralmente m'ha feruto, und'eo d'amore sentomi infiammato" (Pier delle Vigne, Uno piasente sguardo).

${ }^{2}$ L'attenzione dei poeti non si focalizzò particolarmente sulla figura della donna ma, piuttosto, sul fenomeno dell'amore in sé e, di conseguenza, sui processi interni che avvengono nella mente di un individuo. La rappresentazione dell'oggetto d'amore è "un'immagine mentale", "una rappresentazione iconica" o riprodotta "al proprio interno un'immagine fantasmatica", si veda il saggio di Paolo Borsa (Borsa, 2016).

${ }^{3}$ Non è intenzione dell'autrice trarre conclusioni generali riguardanti tutta la narrativa di Camilleri. L'esito di questa ricerca concerne soltanto le due opere in questione, La ripetizione e La Vucciria. 
(2009), La rivoluzione della luna (2013) o Noli me tangere (2016). Un esempio eclatante di questo interesse è il racconto autobiografico intitolato semplicemente Donne, pubblicato nel 2014. La ripetizione, il breve racconto di Camilleri, uno dei più conosciuti e letti scrittori siciliani contemporanei, è solo un pezzo microscopico della sua immensa narrativa, di grande notorietà e di grande diffusione, ma illustra bene anche il suo particolare interesse per l'arte e il rapporto tra parola e immagine, letteratura e pittura, e, persino, l'interazione di tutte le forme d'arte. Questione, questa, che era già stata materia di studio per altri ricercatori (Ladogana, 2016; Marci \& Ruggerini, 2020).

La parte introduttiva di questo lavoro sarà dedicata alla dualità della percezione della donna nel contesto della storia e della cultura siciliana: si può percepire la donna come erede del matriarcato, con il viso prepotente e spaventoso, oppure sottomessa al maschio, resa oggetto e ridotta al ruolo procreativo. Verrà poi evidenziato come, adeguatamente, Andrea Camilleri e Renato Guttuso, nelle opere in questione, rivoltino totalmente le immagini dei loro soggetti e, soprattutto, che essi cercando di delineare la figura della donna, finiscano invece col descrivere l'uomo. A questo fenomeno verrà in seguito dato il nome di "inversione". Effettivamente l'uomo del racconto di Camilleri combatte per la sua identità moderna utilizzando come suo cavallo di battaglia il disprezzo per la femmina, espressione della tradizione patriarcale, compiendo così il simbolico "matricidio". Questo fenomeno è visibile in quella che da ora in poi verrà qui chiamata "decapitazione della Medusa", una nota simbologia presente nella storia dell'arte .

\section{LA TRAMA DE LA RIPETIZIONE}

La trama del racconto La ripetizione è composta da due filoni narrativi paralleli collocati in due periodi distanti tra di loro (nel Seicento e nella contemporaneità). Grazie a questo espediente narrativo, la sospensione tra due epoche che si intrecciano continuamente, viene raccontato

${ }^{4}$ Perseo con la testa di Medusa di Benvenuto Cellini, Scudo con testa di Medusa di Caravaggio, Medusa di Gian Lorenzo Bernini, Medusa di Peter Paul Rubens ecc. 
il destino di due donne: Anna Canzoneri (secentesca) e un'altra Anna (contemporanea) che, il sabato mattina, fa la spesa al mercato Vucciria, dipinto da Renato Guttuso ${ }^{5}$. Le testimonianze di due venditori, Agnozio Calandrino e Michele Mattarulo, di fronte alla Santa Inquisizione, rivelano che Anna Canzoneri fosse una creatura dai poteri sovrannaturali, con una voce forte, la quale, a Calandrino, aveva immobilizzato le gambe. Tutti e due furono affascinati dal suo aspetto attraente, rimanendo poi delusi e gelosi poiché ella al mercato, di fronte a loro, s'incontrava con il suo amante, Antonello Moscato. Calandrino conferma che Anna Canzoneri sia la "donna di fora", cioè una strega. Nel frattempo, Anna contemporanea, come nel quadro, in cerca di carne da acquistare, incontra un bell'uomo, Antonello. Ella è stata vista da un venditore di carne, ed è proprio lui a sospettare che la vicinanza corporale con Antonello abbia provocato in Anna qualche effetto sensuale. Mentre l'Inquisizione, nella realtà secentesca, pronuncia la sua sentenza e condanna la donna "a esser murata viva in una delle secrete dello Steri" (Camilleri, 2011, p. 19), Camilleri propone la sua riflessione sulla situazione della donna sicula dei tempi nostri, la quale sembra non essere cambiata con il passare del tempo ${ }^{6}$.

\section{LA SICILIA COME UNA DONNA - UN QUADRO COMPLESSO}

Il tempo, questo peculiare intreccio di epoche, recitando altresì il ruolo di uno scheletro strutturale della narrazione de La ripetizione, è sicuramente una delle chiavi interpretative importanti da cui bisogna iniziare quest'analisi. Il quadro complessivo dell'evoluzione storica dell'im-

5 Camilleri dichiara apertamente che lo scenario è preso direttamente dal quadro di Guttuso (Camilleri, intervista video su Il quadro nero). Delle somiglianze e differenze tra il quadro con tutti gli abbozzi e il racconto di Camilleri scrive Paulina Malicka (2014).

${ }^{6}$ Lo testimoniano le ultime parole del racconto riguardanti la situazione di Anna contemporanea: "Ora sa che il sò destino è signato, qualisisiasi cosa che possa succediri non potrà cangiarlo. Manco se Peppe la mura viva dintra a 'na cammara" (Camilleri, 2011, p. 19). 
magine della donna sicula (compresa quella mitica) e la sua posizione sociale è cruciale per la comprensione delle briciole di realtà storiche inserite nel testo da Camilleri.

Come riportato da varie fonti storiche e da ricerche archeologiche (Ampolo, 1989; Bosi, 1989a), le popolazioni prima della colonizzazione greca iniziata nell'VIII secolo a. C. in Sicilia furono: i Siculi, i Sicani e gli Elimi. Uno dei culti originali e molto diffusi nella Sicilia centrale ${ }^{7}$, fu quello della Grande Madre Vergine Kore (cf. Martorana, Angelini, \& Greco, 1995, pp. 15-16), associato poi a quello della dea greca Demetra. È anche storicamente accertato che un simbolo presente in Sicilia, ancora prima dell'arrivo dei Greci, fosse la Trinacria. Ciò che distingue la Triscele (o Trischele), simbolo noto ${ }^{8}$ nell'Asia Minore, nell'Attica, nei paesi celtici e l'isola britannica di Man, dalla Trinacria, il simbolo proprio della Sicilia, è la presenza del gorgòneion. Il gorgòneion, cioè la Medusa. La più nota delle tre sorelle Gorgoni, decapitata da Perseo, viene considerata come il simbolo della donna di potere ed è altresì dotata di una certa cattiveria. Il suo ruolo nell'emblema siciliano fu probabilmente quello di spaventare i nemici rappresentando una terribile e minacciosa protettrice. Col passare del tempo, dopo Greci e Romani, si affermò in Sicilia il cristianesimo, probabilmente nel II secolo d. C., come testimoniano le prime sepolture cristiane trovate sull'isola. Una delle prime martiri del cristianesimo in Sicilia fu Sant'Agata, vissuta nel III sec., la patrona di Catania e una delle quattro patrone di Palermo. È proprio grazie a questa apertura mentale verso i diversi culti, all'adorazione popolare delle sante vergini, senza considerare le 70 emanazioni della Madonna, che il culto della donna è sopravvissuto in Sicilia. Il cristianesimo ha portato con sé anche un altro modo di percepire la donna, vista come una persona spiritualmente debole. Come si evince dal Nuovo Testamento ${ }^{9}$, la figura della donna rimane per sempre gravata

7 In particolare si tratta della provincia di Enna, situata nelle zone occupate dai Siculi e Sicani che, in questo territorio, si mescolavano continuamente.

8 Tracce dal VII secolo a. C.

9 "La donna impari in silenzio, con tutta sottomissione. Non concedo a nessuna donna di insegnare, né di dettare legge all'uomo; piuttosto se ne stia in atteggiamento tranquillo. Perché prima è stato formato Adamo e poi Eva; e non fu Adamo ad essere 
dal peso del peccato originale collegato con la sua sessualità e, dunque, ella è obbligata all'obbedienza al maschio, alla sottomissione e al dovere di procreare. La diffusione della fede cristiana e il potere della Chiesa portarono anche ad instaurare in Sicilia una variante più oppressiva dell'Inquisizione, quella spagnola, con sede nel Palazzo Chiaramonte (Steri), a Palermo. Essa nel racconto di Camilleri interroga Anna Canzoneri sulla sua presunta stregoneria.

Secondo Giuseppina Giudici, abituati alle invasioni di popoli provenienti da tutte le direzioni, i siciliani impararono ad essere sospettosi riguardo a qualsiasi struttura statale, stabilita dai governatori di quest' $i-$ sola in ogni periodo storico (Giudici, 1997, p. 13). All'interno di questa mentalità la donna diventa l'elemento centrale della vita familiare. Ella è "la sacerdotessa del focolare", concentrandosi soprattutto sulla cura della famiglia e dei figli, senza mostrare il minimo interesse per l'esterno, inteso come spazio di partecipazione alla vita sociale. La sua continua neutralità sul campo della politica o della religione, infine, la rende più vulnerabile alle leggi emanate con effetti misogini o alle superstizioni. Nel 1734, a Palermo, fu pubblicata un'opera antifemminista $L u$ vivu mortu di Luigi Sarmento ${ }^{10}$, dove la donna viene posta come origine di ogni male. Tuttavia, in questo periodo troviamo anche quattro opere pre-femministe scritte in risposta tra il 1735 ed il 1740 da Genoveffa Bisso dell'Accademia degli Ereini, Dorotea Isabella Bellini Guillon, monaca clarissa, Pietro Pisani da Palermo e Vincenzo di Blasi. Nel suo saggio intitolato La verità manifestata, Apologia in favor delle donne pubblicato nel 1735, Pietro Pisani cerca di affermare che non è stata Eva a commettere il peccato originale, ingannata dal demonio, ma che invece il colpevole sarebbe Adamo che non obbedì al diretto insegnamento di Dio (Pisani, 1735, p. 5). Dal ripasso della storia risulta allora un quadro del mondo femminile siculo, dell'isola, dell'antica dea precerealicola che non ha bisogno dell'uomo in cui si instaurò col tempo

ingannato, ma fu la donna che, ingannata, si rese colpevole di trasgressione. Essa potrà essere salvata partorendo figli, a condizione di perseverare nella fede, nella carità e nella santificazione, con modestia." 1 Tim 1:11-15.

${ }^{10}$ In particolare, per quanto riguarda la traduzione dal siciliano antico, si veda il testo di Santi Correnti (1989). 
una cultura patriarcale, abbinandola spesso incoerentemente all'essenza primaria siciliana. Con l'avvento delle nuove culture e civiltà la figura del maschio, erede di Adamo, e il suo ruolo dominante, ha predominato imponendosi nettamente sulla donna e provocando la collisione di credenze e culture opposte che non sarebbero mai potute andare d'accordo.

\section{IL FENOMENO DE “L'INVERSIONE”}

Così come La ripetizione costituisce un invito alla lettura doppia con il quadro guttusiano, Camilleri stesso incoraggia il lettore alle letture multiple della storia e della cultura. Le realtà, i fatti storici, gli spazi, i simboli, i nomi e il modo di percepire il mondo: tutto è connesso e dura nonostante lo scorrere del tempo. Inoltre, solo in questo modo sarà possibile la riflessione globale sulla condizione della donna nell'universo siciliano. Ecco le parole con le quali l'autore, prendendo spunto dalla lettura del quadro La Vucciria di Guttuso, introduce l'idea della divisione narrativa nel racconto e il suo titolo:

Che cosa viene un secondo dopo che gli sguardi dei due personaggi centrali si incrociano? I due personaggi centrali intendo: il giovane rivolto verso di noi con il maglione giallo e la ragazza di spalle che si sta avvicinando a questo giovane. È inevitabile che da lì a un momento si guarderanno. La domanda è: e se si fossero già guardati? ${ }^{11}$ Se si fossero già guardati non ai giorni nostri ma tanto tempo fa? E quest'incontro è forse la ripetizione di un incontro avvenuto in un altro tempo e un'altra storia, durante l'Inquisizione in Sicilia? (Camilleri, intervista video su Il quadro nero)

Con queste parole Andrea Camilleri introduce anche due personaggi e le loro nuove incarnazioni: Anna e Antonello che potrebbero essere le stesse persone, vissute però in tempi diversi. Anche se, in realtà, è solo il tempo che cambia. Invece Piazza Caracciolo, il nucleo centrale del

11 Nell'altro romanzo intitolato Noli me tangere Camilleri torna alle questioni riguardanti il tocco e lo sguardo. Nelle parole "Ma si sono già toccati!" (Camilleri, 2016, p. 60) che riguardano un'immagine del famoso incontro del Cristo Risorto e la Maddalena, risuona la sua proposta di oltrepassare o reinterpretare le immagini insediate da secoli nelle menti. 
mercato detto Vucciria, rimane lo stesso. Proprio lo spazio, la struttura del racconto La ripetizione, oltre che la composizione della Vucciria di Guttuso, paradossalmente negano il posto centrale del presupposto soggetto del ritratto e della narrazione. Come è possibile evincere, la donna, che avrebbe dovuto essere un soggetto, diventa invece una spettatrice. Non sembra tuttavia una pratica comune ai pittori, ritrarre di spalle la persona-chiave del quadro, come ha fatto Renato Guttuso. La donna guttusiana sembra non partecipare allo spettacolo, quasi come se entrasse in scena dall'esterno. Nel quadro di Guttuso coesistono infatti due sguardi, in accordo con l'analisi di Michel Foucault del quadro Las meninas di Velázquez, quello del creatore della composizione e poi "quello verso il quale [il quadro] si offre" (Foucault \& Panaitescu, 2016, loc 488). Seguendo le parole di Foucault:

il guardante e il guardato si sostituiscono incessantemente l'uno all'altro. Nessuno sguardo è stabile o piuttosto, nel solco neutro dello sguardo, che trafigge perpendicolarmente la tela, soggetto e oggetto, spettatore e modello invertono le loro parti all'infinito (Foucault \& Panaitescu, 2016, loc 286)

Anche nel quadro di Guttuso i ruoli dei soggetti e degli spettatori, come riflessi in uno specchio, si invertono continuamente "l'uno all'altro". Secondo l'analisi proposta da Laura Mulvey (Mulvey, 1989, pp. 14-26), nelle società patriarcali il ruolo dello spettatore è riservato solamente al maschio, in quanto il suo sguardo ${ }^{12}$ ha un carattere "attivo", in opposizione alla passività femminile. Nel racconto La ripetizione, la descrizione del cammino dell'Anna contemporanea in Vucciria si interseca con la narrazione dell'udienza secentesca, dove l'altra Anna viene descritta e accusata di stregoneria. A tutte e due mancano quasi le parole ${ }^{13}$. Esse dicono poco o niente, per questo possono essere considerate (come nel quadro La Vucciria) piuttosto come delle spettatrici in recite messe in scena dai maschi. Anna Canzoneri parla solo alla fine

\footnotetext{
12 Male gaze (sguardo maschile).

13 L'argomento frequente nella letteratura femminile e nelle ricerche femministe, si vedano per es. le opere di Dacia Maraini (La lunga vita di Marianna Ucria) o Antonella Cilento (Lisario o il piacere infinito delle donne).
} 
dell'udienza ${ }^{14}$, dopo essere stata interrogata dall'Inquisitore e prima di sentire la sentenza. Non confessa il peccato, ammette di aver sofferto e di aver goduto, non si pente ma, al contrario, accusa i maschi per le loro false testimonianze. Di nuovo Camilleri inverte i ruoli e la condannata diventa l'accusatore. Accusata di stregoneria l'Anna secentesca, a differenza dell'Anna contemporanea, ha anche il suo cognome: si chiama Canzoneri. Quel cognome non poteva essere stato scelto da Camilleri a caso. Esso è proprio il cognome dell'ultima vittima dell'Inquisizione di Steri (Di Marzo, 1770): il maschio, Antonino Canzoneri (Cansuneri), che fu bruciato vivo il 22 marzo 1732 alle ore 12:30 ${ }^{15}$.

\section{LA DECAPITAZIONE DELLA MEDUSA}

La breve e simultanea analisi del quadro e del racconto ha rivelato il loro legame reciproco e inseparabile. Pertanto, si propone di continuare quest'indagine prestando molta attenzione agli elementi che compongono il dipinto. Esiste una pratica frequente nei laboratori pittorici, fatta per verificare la composizione e per valutare l'armonia e l'estetica del quadro. Quest'ultimo viene girato sottosopra in modo da permettere al pittore-spettatore di distaccarsi dagli oggetti figurativi e concentrarsi soprattutto sulle macchie di colore, sulle linee e le loro relazioni. Dopo "aver girato" La Vucciria una cosa diventa ovvia, cioè che questo quadro ha come suo punto centrale un oggetto che si trova precisamente al centro del quadro: l'ovale bianco della ciotola con le mozzarelle ${ }^{16}$. La seconda cosa che emerge da questo esperimento è l'importanza del corpo del bue, il quale sembra fare da contrappeso alla figura femminile in bianco. Dunque, perché qualche semplice ciotola sarebbe così

14 La stessa è la costruzione del romanzo Noli me tangere (Camilleri, 2016) dove la protagonista scomparsa Laura Garaudo, assente durante la narrazione e punto di interesse di tutte le conversazioni dei maschi, appare solo in uno degli ultimi capitoli per una breve conversazione.

15 Dopo circa tre secoli le sculture dei tre cavalli provenienti dalla scenografia della Norma di Bellini vengono esposte nell'atrio di Palazzo Chiaramonte-Steri. Il loro autore, scultore si chiama: Michele Canzoneri.

16 Il risultato viene confermato dopo aver eseguito le diagonali. 
importante da metterla al centro della tela? L'unica spiegazione logica sarebbe che la ciotola è l'oggetto verso il quale lo spettatore nel primo momento punta i suoi occhi, e così è lo stesso per la donna dipinta di spalle.

Come racconta Fabio Carapezza Guttuso (2011, p. 31), prima di affrontare la sua grande tela, il pittore prepara tre abbozzi. Tranne la tela finale, solo nel primo collage preparativo la testa della donna è diretta in avanti, ma lì non c'è invece la figura dell'uomo. Il maschio appare nell'ultimo schizzo in bianco e nero ma, nello stesso quadro, la donna gira la testa a destra. Sembra che l'idea guttusiana non sia quella di sottolineare l'incontro degli occhi dei due ma che egli, invece, intenda far capire allo spettatore esterno che lei guarda la ciotola, mentre è proprio lui che la guarda di fronte.

Joan Rivière, nel suo lavoro intitolato Womanliness as a Masquerade (Rivière, 1986), pubblicato per la prima volta nel 1929, descrive la spettatrice femminile come una donna partecipante alla mascherata. Shohini Chaudhuri, nel suo libro dedicato alle teoriche del cinema femministe, analizzando il testo della Rivière scrive: "i personaggi femminili che si mascherano vengono spesso puniti, ad esempio femmes fatales che cercano di usurpare l'attività maschile del guardare, o eroine di film horror il cui sguardo terrorizzato è dominato dallo sguardo del mostro" (Chaudhuri, 2006, pp. 41-42, trad. JB). Infatti, la donna in bianco di Guttuso si maschera usurpando il suo sguardo quando entra nel telaio. Similmente al quadro di Velázquez analizzato da Michel Foucault, anche nella Vucciria possiamo identificare due sguardi che si intrecciano, facendo in questo modo emergere anche i due spettatori. La donna in bianco, la femme fatale del racconto di Camilleri guarda la ciotola. L'uomo in giacca che la guarda di fronte, che nel racconto di Camilleri porta il nome Antonello, smaschera, riflesso nello specchio il viso dell'autore del quadro, il pittore Renato Guttuso. Secondo la teorica del cinema, l'americana Mary Ann Doane lo sguardo maschile intersecando lo sguardo femminile nello stesso tempo lo cancella (Chaudhuri, 2006, p. 42). Secondo Laura Mulvey, lo spettatore esterno segue e si identifica sempre con il male gaze, dunque assume solo il punto di vista maschile. In questo contesto, la donna resta per sempre 
identificata come un oggetto sessuale. Infatti, nella Vucciria è inevitabile notare anche la cesellata forma del corpo della donna in bianco con la sua parte posteriore attraente. Sembra un "oggetto" del quadro sul quale tende lo sguardo dello spettatore. La donna, che partecipa all'esibizione delle merci sul mercato, è fisicamente e totalmente incorporata nella realtà e, come altri oggetti, anche nella "natura morta" della Vucciria. Cosa sottolineata anche da Guttuso, che ha contrapposto il corpo del bue alla donna. Werner Haftmann interpreta l'ultima grande versione della Vucciria in olio in questa maniera:

Quest'ultimo dipinto è una visione sintetica del turbolento e chiassoso mercato di Palermo, un'enorme natura morta con oggetti stipati e ammassati gli uni sugli altri, fra i quali si muovono gli acquirenti. Nonostante le presenze umane, i veri protagonisti di questo scenario traboccante sono le cose (Haftmann, 2014, loc 279-283)

In tal modo, l'immagine della donna viene ridotta ad essere anche lei la rappresentazione di "una cosa" - è, piuttosto, un pezzo di "carne" privata della sua spiritualità. Poiché essere carne significa essere esclusi dalla comunione umana, significa essere un animale e, come tale, non avere il privilegio di poter decidere della propria vita.

Anche le donne de La ripetizione di Camilleri sono rappresentate in modo fisico, carnali e sensuali. Vedendo il sangue della carne nel mercato "Anna prova dintra di lei "na sinsazioni stramma di arrimisculiamento che le parte dalla nuca e le arriva ai piedi e che non accapisce di che natura è" (Camilleri, 2011, p. 10). Camminando nella Vucciria,

si senti trimoliare le gamme, l'assuglia 'mproviso un senso di languidizza come doppo 'na fevri e avverte che 'na pica di sudori le vagna il petto e le cosce, proprio come le capitava quanno Peppe, ai primi tempi del matrimonio, appena corcato l'abbrazzava e le faciva accapire la 'ntinzioni (ibid., p. 12)

E quando alla fine incontra al mercato Antonello, bello e sconosciuto, "Anna prova lungo la schina dorsali un addrizzuni di friddo. [...] Il so corpo si fa senza volirlo leggermenti rigido" (ibid., p. 13). 
Così come l'oggettivazione sessuale risulta dal disprezzo, anche l'emanazione della sessualità è la ragione dell'odio diretto alle donne. L'accusa dell'Inquisitore, nella parte riguardante la realtà secentesca, descrive come: "voi in suo potere redutto l'avete e costretto a carnali congiungimenti anche contro natura" (Camilleri, 2011, p. 16). Tuttavia, esso riguarda soltanto il rapporto con Antonello. Nondimeno un altro uomo, quello rifiutato, l'osservatore invidioso, un certo Agnozio Calandrino si sente minacciato. Possiamo leggerlo direttamente dalle parole di Camilleri: "Non sentia quali parole ella dicea a Michele Mattarulo, ma io vedea lui vieppiù pallido in volto diventare e le membra tenere immobili fino a quando con molta difficoltate si mosse siccome sonnambulo" (ibid., 2011, pp. 10-11). Questa donna la si può immaginare come una bellezza straordinaria con i capelli lunghi e brillanti (una visione che allude esplicitamente alla Medusa), piena di confidenza e di orgoglio. Con la voce stupenda, "tal voce che mutato in statua pareami, impastoiate le gambe e più non sapea indove io mi trovava..." (ibid., p. 10) secondo Agnozio Calandrino. Racconta Michele Mattaruolo: "io sentimmi tutto affatare. Mai audita pria aveva voce simile! Ella parlar non parea, ma soavemente cantare come dicono far le sirene co' i naviganti..." (ibid., p. 8). La protagonista viene descritta come una creatura soprannaturale, una sirena che conduce alla morte i marinai ${ }^{17}$. La sua voce - recuperata in un certo senso perché, paradossalmente, resta in silenzio durante quasi tutta la narrazione - costituisce una minaccia costante per il maschio. I due venditori possono solo osservare come essa allarga le braccia per Antonello, lo stringe e lo bacia con amore. In Sicilia,

${ }^{17}$ L'immagine della donna-sirena con una voce ipnotizzante è ricorrente nell'opera di Camilleri dopo il romanzo Maruzza Musumeci appartenente alla Trilogia delle metamorfosi in cui appaiono anche altre figure femminili di origine fantastica Il casellante (2008) e Il sonaglio (2009): "Quella voci era un vento càvudo che ti faciva a picca a picca mancari di piso, po' ti sollevava in aria a leggio a leggio come 'na foglia e ti faciva perdiri dintra al celu" (Camilleri, 2007, p. 57). Si vedano articoli in merito di Vinicio Busacchi (Busacchi, 2019), Simona Demontis (Demontis, 2020), Morena Deriu (Deriu, 2019), Velasco López e Marìa Del Henar (López \& Del Henar, 2018) e Laura Medda (Medda, 2019). 
nella quale, seguendo le parole di Vitaliano Brancati, "i discorsi sulle donne davano un maggior piacere che le donne stesse" (Brancati, 1988 , p. 11) è certo che le donne provocano tanti discorsi tra maschi. Possono osservarle e fare i loro commenti, creare delle storie incredibili immaginate a causa della gelosia e dell'orgoglio maschile ferito. Perché, come dice lei stessa, lei li ha rifiutati: "E tutti i dù più e più volte m'avivano fatto proposte di giacermi secoloro molto concupendomi e promettendomi se io alle voglie loro accondiscendeva l'uno ricchi panni per vestimenta e l'altro un sacchetto di moneta d'oro. Et io sempre a loro erami negata" (Camilleri, 2011, p. 17). Anna Canzoneri è dunque indipendente ed anche autosufficiente, una persona che richiama il suo diritto di decidere della propria vita. Consapevole della propria avvenenza, Anna prosegue con le sue scelte, togliendo così all'uomo la possibilità di esercitare la propria volontà sulla donna (di lei) in quanto considerata subalterna e sdegnata.

Una delle spiegazioni possibili del disprezzo diretto verso il sesso femminile sarebbe proprio quella fornita dalle teorie sulla fobia di natura subcosciente, sulla quale indagano gli psicoanalisti. Concordemente con le parole di Julia Kristeva, il seno della donna (simbolicamente) "possiede tutto quello che egli [il bambino / il maschio] desidera, ha una quantità illimitata di latte e di amore ma che lo tiene per suo gradimento. Questa sensazione aumenta il risentimento e l'odio del bambino e di conseguenza disturba la sua relazione con la madre" (Kristeva, 2006 , p. 3). Come possiamo vedere, la fuga e il panico causata dal sesso femminile si conclude, nel racconto di Camilleri, con il "femminicidio", in cui la donna viene "murata viva in una delle secrete dello Steri" (Camilleri, 2011, p. 19). Melanie Klein ha sottolineato l'invidia come una delle emozioni fondamentali che il neonato sperimenta verso il seno della madre. Da questa gelosia originale del bambino deriverebbero la fobia e il sadismo sub-coscienti. Come scrive Julia Kristeva nel suo libro dedicato alla Klein:

L'universo kleiniano (...) è dominato dalla madre. Questa figura arcaica minaccia e terrorizza con la sua onnipotenza. È così dannosa che è opportuno abbandonarla e farla morire? Non potrebbe trasformarsi? Ma in che cosa? 
L'abbandono necessario della madre è forse un passaggio al padre (Kristeva, 2006, p. 127)

Secondo la Klein, nel neonato coesistono e anche si scontrano il culto della madre con il desiderio del matricidio. Concordemente alle parole di Kristeva:

Entrambi, il culto della madre e il matricidio, sono salvifici. Tuttavia, secondo ogni evidenza, il matricidio lo è di più del culto materno. Senza matricidio, infatti, l'oggetto interno non si costituisce, la fantasia non si costruisce e la riparazione è impossibile (Kristeva, 2006, p. 144)

Così il bambino ha bisogno di distaccarsi, separarsi dalla madre. Kristeva ci riporta alla mente la scena della "decapitazione della Medusa" presente nelle arti occidentali, paragonandola alla castrazione femminile che, simbolicamente, si riferisce alla perdita della madre arcaica. Secondo Sigmund Freud, la testa decapitata della Medusa rappresenta una terrificante donna, una minaccia della vagina dentata e dai fallici capelli/serpenti (Chaudhuri, 2006, p. 99). La testa della Medusa, il gorgòneion, è ciò che distingue la simbolica madre arcaica dei Siciliani - la Trinacria. La decapitazione della Medusa rappresenta, secondo gli psicoanalisti, il simbolico matricidio. Esso è anche presente nell'opera di Andrea Camilleri, dove le donne indipendenti e dominanti da un punto di vista sessuale, proprio per queste loro caratteristiche, vengono percepite dall'uomo come travolgenti e, pertanto, vengono respinte tramite il disprezzo misogino.

\section{CONCLUSIONI}

L'obiettivo del presente lavoro è stato quello di raccogliere le tracce di un possibile ritratto della donna nascoste nella particolare opera ecfrastica La ripetizione di Andrea Camilleri, legata a La Vucciria di Renato Guttuso. A tal fine sono stati individuati i paradigmi con cui lo sguardo dell'uomo siciliano ritrae la donna siciliana. Seguendo il corso della storia, nello scontro di culture opposte, il volto divino della madre preistorica viene percepito come minacciante e travolgente. In questo 
modo, simbolicamente, le dimensioni del viso della donna-madre, secondo il ritratto dell'uomo, sembrano uscire dal telaio ${ }^{18}$ e, perciò, possono essere interpretate come sfuggenti. Quindi, la donna sembra destinata ad essere offuscata e annientata. Il maschio cerca di confermare la sua posizione reale, imposta socialmente e insediata nelle menti della collettività. Colei che doveva essere un soggetto del ritratto, la donna di spalle del celebre quadro di Guttuso - La Vucciria - diventa spettatrice ma, nello stesso momento, essa viene catturata anche dallo sguardo maschile nascosto nella riflessione dentro la tela. Ciò che infine vede l'uomo, il pittore, con un colpo d'occhio verso la donna, è solo il proprio viso riflesso in uno specchio. In questo modo la sua dominazione si dimostra solo illusoria. Tuttavia, il fenomeno dell'inversione di cui sopra è presente anche nel racconto di Camilleri, laddove pare che il soggetto ritratto non siano le donne, bensì gli uomini, raccontando piuttosto di sé e dei problemi della loro identità. Dall'analisi del racconto risulta inoltre che l'essenza delle sue protagoniste femminili è soprattutto il loro corpo, un elemento narrativo, costruito da tessuti e strumenti da sentire, da vivere, per esprimere il proprio "io". I sensi: la vista della donna del quadro e la voce di Anna Canzoneri diventano le armi per riconquistare la loro soggettività. Ma la libera espressione della sessualità e della sensualità femminile solleva soltanto la frustrazione del maschio. Lottando per la propria identità, l'uomo commette il matricidio e la Medusa viene decapitata. Il suo volto, troppo terrificante, che riempie tutto il campo visivo, finalmente cade. La decapitazione della Medusa, la madre arcaica dei siciliani, è infatti una metafora di un rapporto complesso del maschio siciliano con la donna siciliana, basata sul mito e registrata nella ricca iconografia.

18 Mi riferisco alle ricerche sul disegno dei bambini nella diagnosi psicopedagogica conosciute tra l'altro come "test del disegno della famiglia" (in questo caso - della madre) e "test del disegno della figura umana" (si veda: Duksa, 2011). 


\section{BIBLIOGRAFIA}

Antonelli, R. (2008). I Poeti della Scuola siciliana. vol. I: Giacomo da Lentini. Milano: Mondadori.

Ampolo, C. (1989). Italia omnium terrarum parens: la civiltà degli Enotri, Choni, Ausoni, Sanniti, Lucani, Brettii, Sicani, Siculi, Elimi. Milano: Libri Scheiwiller.

Borsa, P. (2016). L'immagine nel cuore e l'immagine nella mente: dal Notaro alla Vita nuova attraverso i due Guidi. In M. Gagliano, Ph. Guérin, \& R. Zanni (Eds.), Les deux Guidi: Guinizzelli et Cavalcanti: Mourir d'aimer et autres ruptures (pp. 75-92). Paris: Presses Sorbonne Nouvelle.

Bosi, R. (1989). Sicani, Siculi, Elimi. Forme di identità, modi di contatto e processi di trasformazione. Milano: Longanesi \& C.

Brancati, V. (1988). Don Giovanni in Sicilia. Milano: Gruppo Editoriale Fabbri, Bompiani, Sonzogno, Etas.

Busacchi, V. (2019). Di una incertezza dell'ermeneutica di Ricœur 'risolta' dalla «trilogia fantastica» di Camilleri. Quaderni camilleriani, 8, Fantastiche e metamorfiche isolitudini, 53-64.

Camilleri, A. (2007). Maruzza Musumeci. Palermo: Sellerio.

Camilleri, A. (2008). Il casellante. Palermo: Sellerio.

Camilleri, A. (2009). Il sonaglio. Palermo: Sellerio.

Camilleri, A. (2011). La ripetizione. Milano: Skira.

Camilleri, A. (2016). Noli me tangere. Milano: Mondadori.

Carapezza Guttuso, F. (2011). Storia di un quadro. Milano: Skira.

Chaudhuri, S. (2006). Feminist Film Theorists: Laura Mulvey, Kaja Silverman, Teresa de Lauretis, Barbara Creed. London e New York: Routledge.

Correnti, S. (1989). Il femminismo precursore della Sicilia del settecento. Catania: Tringale.

Demontis, S. (2020). La Sirena, l'albero e la capra: fantastico Camilleri. Quaderni camilleriani, 11, La seduzione del mito, 60-85.

Deriu, M. (2020). Narrare l'arte, illustrare le parole. Quaderni camilleriani, 12, Parole, musica (e immagini), 15-35.

Duksa, P. (2019). Una Sirena fra testo e ipotesto: leggere Maruzza Musumeci alla luce dell'Odissea. Quaderni camilleriani, 8, Fantastiche e metamorfiche isolitudini, 23-41. 
Fabiano, G. (2019). Profili psicologici di personaggi femminili camilleriani. Quaderni camilleriani, 8, Fantastiche e metamorfiche isolitudini, 64-74.

Fiume, G. (2011). Museo dell'Inquisizione - carcere dei penitenziati c/o Lo Steri-Palazzo Chiaramonte piazza Marina. Retrieved from http://www. provincia.palermo.it/turismo/tesori_d_arte_a_palermo/00008081_Museo_dell_Inquisizione.html.

Foucault, M., \& Panaitescu, E-A. (2016). Le parole e le cose: Un'archeologia delle scienze umane. BUR saggi Rizzoli, edizione digitale.

Giudici, G. (1997). Era o Artemide? Donna e realtà palermitana. Palermo: Quaderni dell'Istituto Siciliano di Studi Politici ed Economici, IV serie.

Haftmann, W. (2014). Guttuso. Milano: Giunti. Prima edizione digitale.

Kristeva, J. (2006). Melanie Klein La madre la follia. Roma: Donzelli.

Ladogana, R. (2016). L'arte italiana del Novecento nel Montalbano di Andrea Camilleri. Medea, vol. II, n. 1. DOI: http://dx.doi.org/10.13125/ medea-2422.

López, V. \& Del Henar, M. (2018). La trilogía de las metamorfosis de A. Camilleri y los Mitos Clásicos. Myrtia, 33, 343-374.

Di Marzo, G. (Ed.). (1770). Diari della città di Palermo dal secolo XVI al XIX pubblicati sui manoscritti della biblioteca comunale. Retrieved from https://archive.org/stream/bibliotecastoriO1unkngoog/bibliotecastori01unkngoog_djvu.txt.

Malicka, P. (2014). La Vuccirìa il quadro itinerante di Renato Guttuso e la sua trasposizione in parole di Andrea Camilleri. In C. Bronowski, \& K. Karp (Eds.), La visione poliprospettica del viaggio in cerca delle identità perdute (pp. 123-148). Toruń: Wydawnictwo Naukowe Uniwersytetu Mikołaja Kopernika.

Marci, G., \& Ruggerini, M. E. (2020). Narrare l'arte, illustrare le parole. Quaderni camilleriani, 12, Parole, musica (e immagini), 15-35.

Martorana, G., Angelini, F., \& Greco, R. (1995). Madonne e Sante di Sicilia. Palermo: Carbone.

Medda, L. (2019). "Torno torno all'àrbolo d'aulivo". Il tragico e il meraviglioso dell'esistenza nei romanzi delle metamorfosi di Andrea Camilleri. Quaderni camilleriani, 8, Fantastiche e metamorfiche isolitudini, 42-52.

Mulvey, L. (1989). Visual and Other Pleasures. London: Palgrave Macmillan UK.

Pisani, P. (1735). La verità manifestata, Palermo: Angelo Felicella. 
Rivière, J. (1986) [1929]. Womanliness as a Masquerade. In V. Burgin, J. Donald, \& C. Kaplan (Eds.), Formations of Fantasy (pp. 35-44). London: Methuen.

Sciascia, L., \& Pitrè, G. (2000). Urla senza suono. Graffiti e disegni dei prigionieri dell'inquisizione. Palermo: Sellerio.

\section{Sitografia}

\section{Camilleri, A., intervista video su Il quadro nero. Retrieved from https://www. youtube.com/watch? $v=6 l T q N a H y G b M$.}

Riassunto: L'argomento principale su cui verte il presente articolo è quello di fornire un'immagine della donna, analizzando un'opera ecfrastica di Andrea Camilleri: La ripetizione, e con l'ausilio de La Vucciria di Renato Guttuso. La dualità della percezione della donna, nella ricca e multimillenaria cultura siciliana, si rispecchia nel testo dello scrittore tramite uno scontro di ruoli e di un'inversione dei posti comunemente assegnati agli individui dalla società. L'analisi qui proposta evidenzia il mutuo scambio degli sguardi, definiti da Laura Mulvey come "maschili" e "femminili", e l'insediamento nel quadro dell'osservatore riflesso dallo specchio, come proposto da Michel Foucault. Le riflessioni degli psicoanalisti, in particolare Julia Kristeva e Melanie Klein, forniscono alcune spiegazioni riguardanti la coesistenza del culto della madre con il desiderio del matricidio. Secondo queste teorie, la figura del maschio/figlio viene sempre gravata da un inconsolabile senso di colpa e da un senso di frustrazione. Ne consegue che i protagonisti maschili del racconto di Camilleri vengano tormentati dal terrore a cui è legato il disprezzo nei confronti del corpo femminile. Infatti, la loro dominazione si dimostra essere solo illusoria: il viso della donna risulta travolgente e minaccioso come quello della Trinacria, la padrona della Sicilia. Infine, il maschio, combattendo per la propria identità, commette il matricidio e la Medusa viene decapitata.

Parole chiave: ritratto, donna, Camilleri, Guttuso, matricidio 